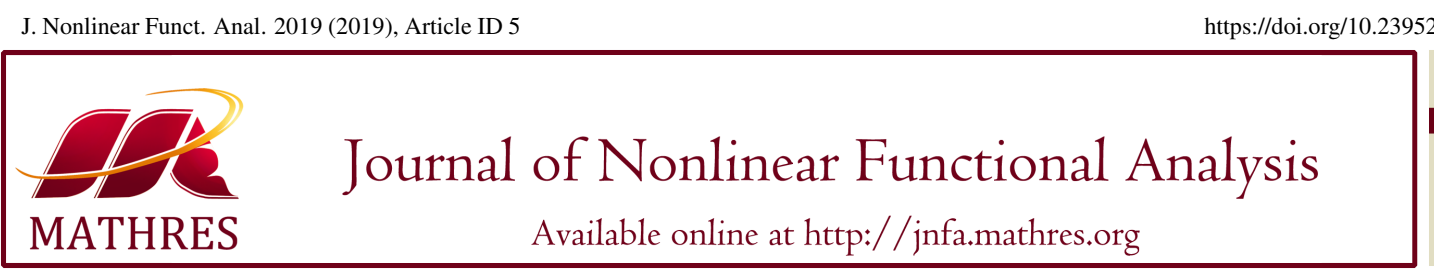

https://doi.org/10.23952/jnfa.2019.5

\title{
POSITIVE SOLUTIONS FOR MULTI POINT IMPULSIVE BOUNDARY VALUE PROBLEMS ON TIME SCALES
}

\author{
İSMAİL YASLAN \\ Department of Mathematics, Pamukkale University, 20070 Denizli, Turkey
}

\begin{abstract}
In this paper, we consider nonlinear second-order multi-point impulsive boundary value problems on time scales. We establish the criteria for the existence of at least one, two and three positive solutions by using the Leray-Schauder fixed point theorem, the Avery-Henderson fixed point theorem and the five functional fixed point theorem, respectively. An example that supports the theoretical results is also provided.
\end{abstract}

Keywords. Boundary Value Problems; Fixed Point Theorems; Impulsive Dynamic Equations; Positive Solutions; Time Scales.

2010 Mathematics Subject Classification. 34B18, 34B37, 34N05.

\section{INTRODUCTION}

The theory of time scales was introduced by Hilger [1] in his Phd thesis in 1988. A result for a dynamic equation contains simultaneously a corresponding result for a differential equation, one for a difference equation, as well as results for other dynamic equations in arbitrary time scales. We refer the reader to the excellent introductory book by Bohner and Peterson [2] and their edited text [3].

Impulsive problems describe processes which experience a sudden change in their states at certain moments. We refer to the books $[4,5,6]$ for the introduction of the theory of impulsive differential equations. The study of impulsive dynamic equations on time scales has also attracted much attention because it provides an unifying structure for differential equations in the continuous cases and finite difference equations in the discrete cases; see, $[7,8,9,10,11,12,13,14,15,16,17,18]$ and references therein.

In 2007, Yaslan [19] discussed the existence of at least one, two and three positive solutions of the nonlinear boundary value problem on time scales:

$$
\left\{\begin{array}{c}
u^{\Delta \nabla}(t)+h(t) f(t, u(t))=0, t \in\left[t_{1}, t_{3}\right] \subset \mathbb{T} \\
u^{\Delta}\left(t_{1}\right)=0, \alpha u\left(t_{3}\right)+\beta u^{\Delta}\left(t_{3}\right)=u^{\Delta}\left(t_{2}\right) .
\end{array}\right.
$$

E-mail address: iyaslan@pau.edu.tr.

Received October 14, 2018; Accepted January 26, 2019.

(C)2019 Journal of Nonlinear Functional Analysis 
In 2014, Karaca, Ozen and Tokmak [20] studied the existence of two or many positive solutions of the nonlinear $p$-Laplacian impulsive boundary value problem on time scales

$$
\left\{\begin{array}{c}
-\left[\phi_{p}\left(u^{\Delta}(t)\right)\right]^{\nabla}=f(t, u(t)), t \in[0,1] \subset \mathbb{T}, t \neq t_{k}, k=1,2, \ldots, m \\
u\left(t_{k}^{+}\right)-u\left(t_{k}^{-}\right)=I_{k}\left(u\left(t_{k}\right)\right) \\
\alpha u(0)-\beta u^{\Delta}(0)=\int_{0}^{1} u(s) \Delta s, u^{\Delta}(1)=0 .
\end{array}\right.
$$

In 2015, Fen and Karaca [21] considered the nonlinear $p$-Laplacian impulsive boundary value problem on time scales

$$
\left\{\begin{array}{c}
-\left[\phi_{p}\left(u^{\Delta}(t)\right)\right]^{\nabla}=f(t, u(t)), t \in[0,1] \subset \mathbb{T}, t \neq t_{k}, k=1,2, \ldots, n \\
u\left(t_{k}^{+}\right)-u\left(t_{k}^{-}\right)=I_{k}\left(u\left(t_{k}\right)\right) \\
u^{\Delta}(0)=0, \quad \alpha u(1)+\beta u^{\Delta}(1)=\sum_{i=1}^{m-2} a_{i} u\left(\xi_{i}\right)
\end{array}\right.
$$

and established criteria for the existence of at least one positive solution to the problem.

In this paper, we consider the following boundary value problem (BVP)

$$
\left\{\begin{array}{c}
y^{\Delta \nabla}(t)+h(t) f(t, y(t))=0, t \in[a, b] \subset \mathbb{T}^{*}, \\
y\left(t_{k}^{+}\right)-y\left(t_{k}^{-}\right)=I_{k}\left(y\left(t_{k}\right)\right), t \neq t_{k}, k=1,2, \ldots, m, \\
y^{\Delta}(a)=0, \quad \alpha y(b)+\beta y^{\Delta}(b)=\sum_{i=1}^{n-2} y^{\Delta}\left(\mu_{i}\right), n \geq 3
\end{array}\right.
$$

where $\mathbb{T}^{*}=\mathbb{T}^{k} \cap \mathbb{T}_{k}, 0 \leq a<t_{1}<\ldots<t_{m} \leq \rho(b), \mu_{i} \in(a, b) \cap \mathbb{T}^{*}(i=1,2, \ldots, n-2)$ with $a<\mu_{1}<$ $\ldots<\mu_{n-2}<b$ and

(H1) $h \in C_{l d}([a, b],[0, \infty))$ and does not vanish identically on any closed subinterval of $[a, b]$;

(H2) $f \in C([a, b] \times[0, \infty),[0, \infty))$;

(H3) $I_{k} \in C\left(\mathbb{R}, \mathbb{R}^{-}\right), t_{k} \in[a, b]$ and $y\left(t_{k}^{+}\right)=\lim _{h \rightarrow 0} y\left(t_{k}+h\right), y\left(t_{k}^{-}\right)=\lim _{h \rightarrow 0} y\left(t_{k}-h\right)$ represent the right and left limits of $y(t)$ at $t=t_{k}, k=1, \ldots, m$.

In this paper, conditions for the existence of at least one positive solutions to BVP (1.1) are first discussed by using the Leray-Schauder fixed-point theorem. Then, we use the Avery-Henderson fixed point theorem to show that the existence of at least two positive solutions for BVP (1.1). Finally, existence result of at least three positive solutions of BVP (1.1) is established as a result of five functionals fixed-point theorem.

\section{PRELIMINARIES}

We now state and prove several lemmas which are needed later.

Lemma 2.1. Assume (H3) holds and $\alpha \neq 0$. If $\omega \in C_{l d}[a, b]$ and $\omega(t) \geq 0$ for $t \in[a, b]$, then $y(t)$ is a solution of the following $B V P$

$$
\left\{\begin{array}{c}
y^{\Delta \nabla}(t)+\omega(t)=0, t \in[a, b] \subset \mathbb{T}^{*} \\
y\left(t_{k}^{+}\right)-y\left(t_{k}^{-}\right)=I_{k}\left(y\left(t_{k}\right)\right), t \neq t_{k}, k=1,2, \ldots, m \\
y^{\Delta}(a)=0, \quad \alpha y(b)+\beta y^{\Delta}(b)=\sum_{i=1}^{n-2} y^{\Delta}\left(\mu_{i}\right), \quad n \geq 3
\end{array}\right.
$$


if and only if $y(t)$ is a solution of the following integral equation

$$
y(t)=\int_{a}^{b}\left(\frac{\beta}{\alpha}+r-a\right) \omega(r) \nabla r-\frac{1}{\alpha} \sum_{i=1}^{n-2} \int_{a}^{\mu_{i}} \omega(r) \nabla r-\sum_{t<t_{k}<b} I_{k}\left(y\left(t_{k}\right)\right)-\int_{a}^{t}(r-a) \omega(r) \nabla r .
$$

Proof. Suppose that $y$ is a solution of BVP (2.1). Then, $y^{\Delta \nabla}(t)=-\omega(t)$ for $t \in[a, b]$. A nabla integration from $a$ to $t$ of both sides of the above equality yields

$$
y^{\Delta}(t)-y^{\Delta}(a)=-\int_{a}^{t} \omega(r) \nabla r \text {, i.e., } y^{\Delta}(t)=-\int_{a}^{t} \omega(r) \nabla r .
$$

Integrating above equality from $a$ to $t$, we get

$$
y(t)-y(a)=-\int_{a}^{t} \int_{a}^{s} \omega(r) \nabla r \Delta s+\sum_{a<t_{k}<t} I_{k}\left(y\left(t_{k}\right)\right) .
$$

It follows that

$$
\begin{aligned}
y(t) & =y(a)-\int_{a}^{t} \int_{a}^{r} \omega(r) \Delta s \nabla r+\sum_{a<t_{k}<t} I_{k}\left(y\left(t_{k}\right)\right) \\
& =y(a)-\int_{a}^{t}(r-a) \omega(r) \nabla r+\sum_{a<t_{k}<t} I_{k}\left(y\left(t_{k}\right)\right) .
\end{aligned}
$$

From the second boundary condition, we obtain

$$
y(a)=\int_{a}^{b}\left(\frac{\beta}{\alpha}+r-a\right) \omega(r) \nabla r-\frac{1}{\alpha} \sum_{i=1}^{n-2} \int_{a}^{\mu_{i}} \omega(r) \nabla r-\sum_{a<t_{k}<b} I_{k}\left(y\left(t_{k}\right)\right) .
$$

Thus,

$$
y(t)=\int_{a}^{b}\left(\frac{\beta}{\alpha}+r-a\right) \omega(r) \nabla r-\frac{1}{\alpha} \sum_{i=1}^{n-2} \int_{a}^{\mu_{i}} \omega(r) \nabla r-\sum_{t<t_{k}<b} I_{k}\left(y\left(t_{k}\right)\right)-\int_{a}^{t}(r-a) \omega(r) \nabla r .
$$

Conversely, it is easy to show that $y(t)$ in (2.2) satisfies (2.1). This completes the proof.

Lemma 2.2. If $\alpha>0, \beta \geq n-2, \omega \in C_{l d}[a, b]$ and $\omega(t) \geq 0$ for $t \in[a, b]$, then the unique solution of $B V P(2.1)$ satisfies $y(t) \geq 0$ for $t \in[a, b]$. 
Proof. Since $y^{\Delta}(t)=-\int_{a}^{t} \omega(r) \nabla r \leq 0, y$ is non-increasing on [a,b]. Therefore, if $y(b) \geq 0$, then $y(t) \geq 0$ for $t \in[a, b]$.

$$
\begin{aligned}
y(b) & =\frac{\beta}{\alpha} \int_{a}^{b} \omega(r) \nabla r-\frac{1}{\alpha} \int_{a}^{\mu_{1}} \omega(r) \nabla r-\frac{1}{\alpha} \int_{a}^{\mu_{2}} \omega(r) \nabla r-\cdots-\frac{1}{\alpha} \int_{a}^{\mu_{n-2}} \omega(r) \nabla r \\
& \geq \frac{\beta}{\alpha} \int_{a}^{\mu_{n-2}} \omega(r) \nabla r-\frac{n-2}{\alpha} \int_{a}^{\mu_{n-2}} \omega(r) \nabla r \\
& =\frac{\beta-n+2}{\alpha} \int_{a}^{\mu_{n-2}} \omega(r) \nabla r \\
& \geq 0 .
\end{aligned}
$$

This completes the proof.

Let

$$
\begin{aligned}
E= & \left\{y:[a, b] \rightarrow \mathbb{R} \text { is continuous at } t \neq t_{k} \text { left continuous at the points } t_{k},\right. \\
& \text { for which } \left.y\left(t_{k}^{-}\right) \text {and } y\left(t_{k}^{+}\right) \text {exist with } y\left(t_{k}^{-}\right)=y\left(t_{k}\right), k=1, \ldots, m .\right\},
\end{aligned}
$$

which is a Banach space with the norm $\|y\|=\sup _{t \in[a, b]}|y(t)|$. Define the cone $P \subset E$ by

$$
P=\left\{y \in E: y \text { is concave, non-decreasing and nonnegative on }[a, b], y^{\Delta}(a)=0\right\} .
$$

Lemma 2.3. Assume $\alpha>0, \beta \geq n-2$. If $y \in P$, then $y(t)$ in (2.2) satisfies

$$
y(t) \geq \frac{b-t}{b}\|y\|, t \in[a, b] \subset \mathbb{T}^{*} .
$$

Proof. Since $y(t)$ is nonincreasing on $[a, b]$, we have $\|y\|=y(a)$. Assume $g(t)=y(t)-\frac{b-t}{b}\|y\|$ for $t \in[a, b] \subset \mathbb{T}^{*}$. From the fact that $g^{\Delta \nabla}(t)=y^{\Delta \nabla}(t) \leq 0$, we know that the graph of $g$ is concave on $[a, b]$. We get

and

$$
g(a)=\frac{a}{b} y(a) \geq 0
$$

$$
g(b)=y(b) \geq 0 .
$$

Therefore, we have $g(t) \geq 0$ for $t \in[a, b]$ from the concavity of $g$. Thus, we obtain $y(t) \geq \frac{b-t}{b}\|y\|$ for $t \in[a, b] \subset \mathbb{T}^{*}$.

By Lemma 2.1, the solutions of BVP (1.1) are the fixed points of the operator $A$ defined by

$$
\begin{aligned}
A y(t) & =\int_{a}^{b}\left(\frac{\beta}{\alpha}+s-a\right) h(s) f(s, y(s)) \nabla s-\frac{1}{\alpha} \sum_{i=1}^{n-2} \int_{a}^{\mu_{i}} h(s) f(s, y(s)) \nabla s-\sum_{t<t_{k}<b} I_{k}\left(y\left(t_{k}\right)\right) \\
& -\int_{a}^{t}(s-a) h(s) f(s, y(s)) \nabla s .
\end{aligned}
$$


Now, we state the fixed point theorems to prove the main results of this paper.

Theorem 2.4 ([22]). (Leray-Schauder Fixed Point Theorem) Let $E$ be a Banach space, and let $A: E \rightarrow E$ be a completely continuous operator. If the set $\{x \in E: x=\lambda A x, 0<\lambda<1\}$ is bounded, then $A$ has at least one fixed point in the closed $T \subset E$, where

$$
T=\{x \in E:\|x\| \leq R\}, R=\sup \{\|x\|: x=\lambda A x, 0<\lambda<1\} .
$$

Theorem 2.5 ([23]). (Avery-Henderson Fixed Point Theorem) Let $P$ be a cone in a real Banach space E. Set

$$
P(\phi, r)=\{u \in P: \phi(u)<r\} .
$$

Let $\eta$ and $\phi$ be increasing, nonnegative continuous functionals on $P$. Let $\theta$ be a nonnegative continuous functional on $P$ with $\theta(0)=0$ such that, for some positive constants $r$ and $M$,

$$
\phi(u) \leq \theta(u) \leq \eta(u) \text { and }\|u\| \leq M \phi(u)
$$

for all $u \in \overline{P(\phi, r)}$. Suppose that there exist positive numbers $p<q<r$ such that

$$
\theta(\lambda u) \leq \lambda \theta(u), \text { for all } 0 \leq \lambda \leq 1 \text { and } u \in \partial P(\theta, q)
$$

If $A: \overline{P(\phi, r)} \rightarrow P$ is a completely continuous operator satisfying

(i) $\phi(A u)>r$ for all $u \in \partial P(\phi, r)$,

(ii) $\theta(A u)<q$ for all $u \in \partial P(\theta, q)$,

(iii) $P(\eta, p) \neq \emptyset$ and $\eta(A u)>p$ for all $u \in \partial P(\eta, p)$,

then $A$ has at least two fixed points $u_{1}$ and $u_{2}$ such that

$$
p<\eta\left(u_{1}\right) \text { with } \theta\left(u_{1}\right)<q \text { and } q<\theta\left(u_{2}\right) \text { with } \phi\left(u_{2}\right)<r .
$$

Now, we will present the five functionals fixed point theorem. Let $\varphi, \eta, \theta$ be nonnegative continuous convex functionals on the cone $P$, and $\gamma, \psi$ nonnegative continuous concave functionals on the cone $\mathrm{P}$. For nonnegative numbers $h, p, q, r$ and $d$, define the following convex sets:

$$
\begin{gathered}
P(\varphi, r)=\{x \in P: \varphi(x)<r\}, \\
P(\varphi, \gamma, p, r)=\{x \in P: p \leq \gamma(x), \varphi(x) \leq r\}, \\
Q(\varphi, \eta, d, r)=\{x \in P: \eta(x) \leq d, \varphi(x) \leq r\}, \\
P(\varphi, \theta, \gamma, p, q, r)=\{x \in P: p \leq \gamma(x), \theta(x) \leq q, \varphi(x) \leq r\}, \\
Q(\varphi, \eta, \psi, h, d, r)=\{x \in P: h \leq \psi(x), \eta(x) \leq d, \varphi(x) \leq r\} .
\end{gathered}
$$

Theorem 2.6 ([24]). (Five Functionals Fixed Point Theorem) Let P be a cone in a real Banach space E. Suppose that there exist nonnegative numbers $r$ and $M$, nonnegative continuous concave functionals $\gamma$ and $\psi$ on $P$, and nonnegative continuous convex functionals $\varphi, \eta$ and $\theta$ on $P$ such that

$$
\gamma(x) \leq \eta(x),\|x\| \leq M \varphi(x), \forall x \in \overline{P(\varphi, r)} .
$$

Suppose that $A: \overline{P(\varphi, r)} \rightarrow \overline{P(\varphi, r)}$ is a completely continuous and there exist nonnegative numbers $h, p, k, q$, with $0<p<q$ such that

(i) $\{x \in P(\varphi, \theta, \gamma, q, k, r): \gamma(x)>q\} \neq \emptyset$ and $\gamma(A x)>q$ for $x \in P(\varphi, \theta, \gamma, q, k, r)$,

(ii) $\{x \in Q(\varphi, \eta, \psi, h, p, r): \eta(x)<p\} \neq \emptyset$ and $\eta(A x)<p$ for $x \in Q(\varphi, \eta, \psi, h, p, r)$,

(iii) $\gamma(A x)>q$, for $x \in P(\varphi, \gamma, q, r)$, with $\theta(A x)>k$, 
(iv) $\eta(A x)<p$, for $x \in Q(\varphi, \eta, p, r)$, with $\psi(A x)<h$.

Then $A$ has at least three fixed points $x_{1}, x_{2}, x_{3} \in \overline{P(\varphi, r)}$ such that

$$
\eta\left(x_{1}\right)<p, \quad \gamma\left(x_{2}\right)>q, \quad \eta\left(x_{3}\right)>p \quad \text { with } \gamma\left(x_{3}\right)<q .
$$

\section{Main Results}

We will apply the Leray-Schauder Fixed Point Theorem to the existence of at least one positive solution for BVP (1.1).

Theorem 3.1. Assume (H1)-(H3) hold and $\alpha>0, \beta \geq n-2$. If there exist numbers $c_{k}$ such that $\left|I_{k}\left(y\left(t_{k}\right)\right)\right| \leq c_{k}$, for $k=1, \ldots, m$, then $B V P(1.1)$ has at least one positive solution.

Proof. For all $y \in P$, we know from (H1), (H2), the definition of $A$ and the proof of Lemma 2.2 that $(A y)(t) \geq 0,(A y)^{\Delta}(t) \geq 0,(A y)^{\Delta \nabla}(t) \leq 0$ and $(A y)^{\Delta}(a)=0$. So $A$ is an operator from $P$ to $P$. It is easy to show that $A: P \rightarrow P$ is completely continuous by using the Arzela-Ascoli theorem. We denote $N(A):=$ $\{y \in P: y=\lambda A y, 0<\lambda<1\}$. Now we show that the set $N(A)$ is bounded. If $T=\{y \in P:\|y\| \leq R\}$ and $R=\sup \{\|y\|: y=\lambda A y, 0<\lambda<1\}$, then

$$
|y(t)|=\lambda|A y(t)| \leq \lambda \sup _{t \in[a, b], y \in T} f(t, y(t))\left\{\left(\frac{\beta+n-2}{\alpha}+b-a\right) \int_{a}^{b} h(s) \nabla s\right\}+\lambda \sum_{k=1}^{m} c_{k}
$$

for all $y \in N(A)$. Then, we obtain $N(A)$ is bounded from (H1) and (H2). By Theorem 2.4, BVP (1.1) has at least one positive solution.

Define the constants

$$
\begin{aligned}
& K:=\frac{\alpha}{\beta-n+2}\left(\int_{a}^{\mu_{n-2}} h(s) \nabla s\right)^{-1}, \\
& L:=\left(\int_{a}^{b}\left(\frac{\beta}{\alpha}+s-a\right) h(s) \nabla s\right)^{-1}
\end{aligned}
$$

and

$$
M:=\left(\int_{a}^{\mu_{n-2}}\left(\frac{\beta-n+2}{\alpha}+s-a\right) h(s) \nabla s\right)^{-1} .
$$

Now, we use the Avery-Henderson fixed point theorem to prove the next theorem.

Theorem 3.2. Assume (H1)-(H3) hold and $\alpha>0, \beta>n-2$. Suppose there exist numbers $0<p<q<r$ such that $\sum_{\mu_{n-2}<t_{k}<b} I_{k}\left(y\left(t_{k}\right)\right) \geq-\frac{q}{2}$ and the function $f$ satisfies the following conditions:

(i) $f(s, y)>r K$ for $(s, y) \in\left[a, \mu_{n-2}\right] \times\left[r, \frac{b r}{b-\mu_{n-2}}\right]$,

(ii) $f(s, y)<\frac{q L}{2}$ for $(s, y) \in[a, b] \times\left[0, \frac{b q}{b-\mu_{n-2}}\right]$,

(iii) $f(s, y)>p M$ for $(s, y) \in\left[a, \mu_{n-2}\right] \times\left[\frac{b-\mu_{n-2}}{b} p, p\right]$,

where $K, L$ and $M$ are defined in (3.1), (3.2) and (3.3), respectively. Then BVP (1.1) has at least two positive solutions $y_{1}$ and $y_{2}$ such that

$$
y_{1}(a)>p \text { with } y_{1}\left(\mu_{n-2}\right)<q \text { and } q<y_{2}\left(\mu_{n-2}\right)<r .
$$


Proof. Define the cone $P$ as in (2.3). We know that $A: P \rightarrow P$ is completely continuous by using ArzelaAscoli theorem. Let the nonnegative increasing continuous functionals $\phi, \theta$ and $\eta$ be defined on the cone $P$ by $\phi(y):=y\left(\mu_{n-2}\right), \theta(y):=y\left(\mu_{n-2}\right)$ and $\eta(y):=y(a)$. For each $y \in P$, we have $\phi(y)=\theta(y) \leq \eta(y)$. Using (2.4), we have

$$
\|y\| \leq \frac{b}{b-\mu_{n-2}} \phi(y) .
$$

Moreover, $\theta(0)=0$. For all $y \in P, \lambda \in[0,1]$, we have $\theta(\lambda y)=\lambda \theta(y)$. We now verify that the remaining conditions of Theorem 2.5 hold.

If $y \in \partial P(\phi, r)$, we see from (2.4) that

$$
r=y\left(\mu_{n-2}\right) \leq y(s) \leq\|y\| \leq \frac{b r}{b-\mu_{n-2}}
$$

for $s \in\left[a, \mu_{n-2}\right]$. Then, by using hypothesis $(i)$, we find

$$
\begin{aligned}
\phi(A y) & =\int_{a}^{b}\left(\frac{\beta}{\alpha}+s-a\right) h(s) f(s, y(s)) \nabla s-\frac{1}{\alpha} \sum_{i=1}^{n-2} \int_{a}^{\mu_{i}} h(s) f(s, y(s)) \nabla s \\
& -\sum_{\mu_{n-2}<t_{k}<b} I_{k}\left(y\left(t_{k}\right)\right)-\int_{a}^{\mu_{n-2}}(s-a) h(s) f(s, y(s)) \nabla s \\
& \geq \frac{\beta}{\alpha} \int_{a}^{\mu_{n-2}} h(s) f(s, y(s)) \nabla s-\frac{n-2}{\alpha} \int_{a}^{\mu_{n-2}} h(s) f(s, y(s)) \nabla s \\
& >\frac{\beta-n+2}{\alpha} \int_{a}^{\mu_{n-2}} h(s) r K \nabla s \\
& =r .
\end{aligned}
$$

Thus condition $(i)$ of Theorem 2.5 is satisfied.

If $y \in \partial P(\theta, q)$, we have from (2.4) that

$$
0 \leq y(s) \leq\|y\| \leq \frac{b q}{b-\mu_{n-2}}
$$

for $s \in[a, b]$. Then, from hypothesis $(i i)$, we get

$$
\begin{aligned}
\theta(A y) & =\int_{a}^{b}\left(\frac{\beta}{\alpha}+s-a\right) h(s) f(s, y(s)) \nabla s-\frac{1}{\alpha} \sum_{i=1}^{n-2} \int_{a}^{\mu_{i}} h(s) f(s, y(s)) \nabla s \\
& -\sum_{\mu_{n-2}<t_{k}<b} I_{k}\left(y\left(t_{k}\right)\right)-\int_{a}^{\mu_{n-2}}(s-a) h(s) f(s, y(s)) \nabla s \\
& <\int_{a}^{b}\left(\frac{\beta}{\alpha}+s-a\right) h(s) \frac{q L}{2} \nabla s-\sum_{\mu_{n-2}<t_{k}<b} I_{k}\left(y\left(t_{k}\right)\right) \\
& \leq q .
\end{aligned}
$$

Hence, condition (ii) of Theorem 2.5 holds. 
Since $0 \in P$ and $p>0$, we have $P(\eta, p) \neq \emptyset$. If $y \in \partial P(\eta, p)$, we obtain from (2.4) that

$$
\frac{b-\mu_{n-2}}{b} p \leq y\left(\mu_{n-2}\right) \leq y(s) \leq y(a)=p
$$

for $s \in\left[a, \mu_{n-2}\right]$ Thus, by hypothesis (iii), we obtain

$$
\begin{aligned}
\eta(A y) & =\int_{a}^{b}\left(\frac{\beta}{\alpha}+s-a\right) h(s) f(s, y(s)) \nabla s-\frac{1}{\alpha} \sum_{i=1}^{n-2} \int_{a}^{\mu_{i}} h(s) f(s, y(s)) \nabla s-\sum_{a<t_{k}<b} I_{k}\left(y\left(t_{k}\right)\right) \\
& \geq \int_{a}^{\mu_{n-2}}\left(\frac{\beta-n+2}{\alpha}+s-a\right) h(s) f(s, y(s)) \nabla s \\
& >p .
\end{aligned}
$$

Since all the conditions of Theorem 2.5 are fulfilled, we conclude that BVP (1.1) has at least two positive solutions $y_{1}$ and $y_{2}$ such that $y_{1}(a)>p$ with $y_{1}\left(\mu_{n-2}\right)<q$ and $q<y_{2}\left(\mu_{n-2}\right)<r$. This completes the proof.

Now, we apply the five functionals fixed point theorem to the existence of at least three positive solutions for BVP (1.1).

Theorem 3.3. Assume (H1)-(H3) hold and $\alpha>0, \beta \geq n-2$. Suppose that there exist constants $0<p<$ $q<\frac{q b}{b-\mu_{n-2}}<r$ such that $\sum_{a<t_{k}<b} I_{k}\left(y\left(t_{k}\right)\right) \geq-\frac{p}{2}$ and the function $f$ satisfies the following conditions:

(i) $f(s, y) \leq \frac{r L}{2}$ for $(s, y) \in[a, b] \times[0, r]$,

(ii) $f(s, y)>q N$ for $(s, y) \in\left[\mu_{n-2}, b\right] \times\left[q, \frac{q b}{b-\mu_{n-2}}\right]$,

(iii) $f(s, y)<\frac{p L}{2}$ for $(s, y) \in[a, b] \times[0, p]$,

where $N=\frac{\alpha}{\beta}\left(\int_{\mu_{n-2}}^{b} h(s) \nabla s\right)^{-1}$ and L is defined in (3.2). Then BVP (1.1) has at least three positive solutions $y_{1}, y_{2}$ and $y_{3}$ satisfying

$$
y_{1}(a)<p<y_{3}(a), y_{3}(b)<q<y_{2}(b) .
$$

Proof. Define the cone $P$ as in (2.3) and define these maps $\gamma(y)=\psi(y)=y(b), \theta(y)=y\left(\mu_{n-2}\right), \varphi(y)=$ $\eta(y)=y(a)$. Then $\gamma$ and $\psi$ are nonnegative continuous concave functionals on $P$, and $\varphi, \eta$ and $\theta$ are nonnegative continuous convex functionals on $P$. Let $P(\varphi, r), P(\varphi, \gamma, p, r), Q(\varphi, \eta, d, r), P(\varphi, \theta, \gamma, p, q, r)$ and $Q(\varphi, \eta, \psi, h, d, r)$ be defined by (2.5). It is clear that $\gamma(y) \leq \eta(y)$ and $\|y\|=\varphi(y)$ for all $y \in \overline{P(\varphi, r)}$.

If $y \in \overline{P(\varphi, r)}$, then $y(s) \in[0, r]$ for all $s \in[a, b]$. By hypothesis $(i)$, we find

$$
\begin{aligned}
\varphi(A y) & =\int_{a}^{b}\left(\frac{\beta}{\alpha}+s-a\right) h(s) f(s, y(s)) \nabla s-\frac{1}{\alpha} \sum_{i=1}^{n-2} \int_{a}^{\mu_{i}} h(s) f(s, y(s)) \nabla s-\sum_{a<t_{k}<b} I_{k}\left(y\left(t_{k}\right)\right) \\
& \leq \int_{a}^{b}\left(\frac{\beta}{\alpha}+s-a\right) h(s) \frac{r L}{2} \nabla s-\sum_{a<t_{k}<b} I_{k}\left(y\left(t_{k}\right)\right) \\
& <r .
\end{aligned}
$$

Then, $A: \overline{P(\varphi, r)} \rightarrow \overline{P(\varphi, r)}$.

Now we verify that the remaining conditions of Theorem 2.6. 
Let $y_{1}=q+\varepsilon_{1}$ such that $0<\varepsilon_{1}<\left(\frac{b}{b-\mu_{n-2}}-1\right) q$. Since

and

$$
\begin{gathered}
\gamma\left(y_{1}\right)=q+\varepsilon_{1}>q, \\
\theta\left(y_{1}\right)=q+\varepsilon_{1}<\frac{q b}{b-\mu_{n-2}}
\end{gathered}
$$

we obtain

$$
\varphi\left(y_{1}\right)=q+\varepsilon_{1}<\frac{q b}{b-\mu_{n-2}}<r
$$

$$
\left\{y \in P\left(\varphi, \theta, \gamma, q, \frac{q b}{b-\mu_{n-2}}, r\right): \gamma(y)>q\right\} \neq \emptyset .
$$

If $y \in P\left(\varphi, \theta, \gamma, q, \frac{q b}{b-\mu_{n-2}}, r\right)$, then we have $q \leq y(s) \leq \frac{q b}{b-\mu_{n-2}}$ for all $s \in\left[\mu_{n-2}, b\right]$. By the hypothesis (ii), we obtain

$$
\begin{aligned}
\gamma(A y) & =\frac{\beta}{\alpha} \int_{a}^{b} h(s) f(s, y(s)) \nabla s-\frac{1}{\alpha} \sum_{i=1}^{n-2} \int_{a}^{\mu_{i}} h(s) f(s, y(s)) \nabla s \\
& \geq \frac{\beta-n+2}{\alpha} \int_{a}^{\mu_{n-2}} h(s) f(s, y(s)) \nabla s+\frac{\beta}{\alpha} \int_{\mu_{n-2}}^{b} h(s) f(s, y(s)) \nabla s \\
& >\frac{\beta}{\alpha} \int_{\mu_{n-2}}^{b} h(s) q N \nabla s \\
& =q .
\end{aligned}
$$

Thus, condition $(i)$ of Theorem 2.6 is fulfilled.

Let $y_{2}=p-\varepsilon_{2}$ such that $0<\varepsilon_{2}<\left(1-\frac{b}{b-\mu_{n-2}}\right) p$. Since

$$
\begin{gathered}
\eta\left(y_{2}\right)=p-\varepsilon_{2}<p, \\
\psi\left(y_{2}\right)=p-\varepsilon_{2}>\frac{b p}{b-\mu_{n-2}}
\end{gathered}
$$

and

$$
\varphi\left(y_{2}\right)=p-\varepsilon_{2}<r
$$

we find

$$
\left\{y \in Q\left(\varphi, \eta, \psi, \frac{b p}{b-\mu_{n-2}}, p, r\right): \eta(y)<p\right\} \neq \emptyset .
$$

If $y \in Q\left(\varphi, \eta, \psi, \frac{b p}{b-\mu_{n-2}}, p, r\right)$, then we obtain $0 \leq y(s) \leq p$ for $s \in[a, b]$. Hence,

$$
\begin{aligned}
\eta(A y) & =\int_{a}^{b}\left(\frac{\beta}{\alpha}+s-a\right) h(s) f(s, y(s)) \nabla s-\frac{1}{\alpha} \sum_{i=1}^{n-2} \int_{a}^{\mu_{i}} h(s) f(s, y(s)) \nabla s-\sum_{a<t_{k}<b} I_{k}\left(y\left(t_{k}\right)\right) \\
& <\int_{a}^{b}\left(\frac{\beta}{\alpha}+s-a\right) h(s) \frac{p L}{2} \nabla s-\sum_{a<t_{k}<b} I_{k}\left(y\left(t_{k}\right)\right) \\
& \leq p
\end{aligned}
$$

by the hypothesis $(i i i)$. It follows that condition (ii) of Theorem 2.6 holds. The conditions (iii) and (iv) of Theorem 2.6 is clear. This completes the proof. 
Example 3.4. Let $\mathbb{T}=\mathbb{R}$. Consider the following boundary value problem:

$$
\left\{\begin{array}{c}
y^{\Delta \nabla}(t)+\frac{y^{2} t}{y^{2}+1}=0, t \neq \frac{9}{2}, t \in[1,5] \subset \mathbb{T} \\
y\left(\frac{9}{2}^{+}\right)-y\left(\frac{9}{2}^{-}\right)=-0.001 \\
y^{\Delta}(1)=0, \quad y(5)+5 y^{\Delta}(5)=y^{\Delta}(3)+y^{\Delta}(4) .
\end{array}\right.
$$

If we take $p=0.71, q=0.8$ and $r=22$, then all the conditions in Theorem 3.2 are satisfied. Thus, the BVP has at least two positive solutions $y_{1}$ and $y_{2}$ satisfying $y_{1}(1)>0.71$ with $y_{1}(4)<0.8$ and $0.8<y_{2}(4)<22$.

If we take $p=0.0055, q=20$ and $r=180$, then all the conditions in Theorem 3.3 are satisfied. Thus, the BVP has at least three positive solutions $y_{1}, y_{2}$ and $y_{3}$ such that

$$
y_{1}(1)<0.0055<y_{3}(1), y_{3}(5)<20<y_{2}(5) .
$$

\section{REFERENCES}

[1] S. Hilger, Analysis on measure chains-A unified approach to continuous and discrete calculus, Results Math. 18 (1990), 18-56.

[2] M. Bohner, A. Peterson, Dynamic Equations on Time Scales: An Introduction with Applications, Birkhäuser, Boston, 2001.

[3] M. Bohner, A. Peterson, Advances in Dynamic Equations on Time Scales, Birkhäuser, Boston, 2003.

[4] M. Benchohra, J. Henderson, S. Ntouyas, Impulsive Differential Equations and Inclusions, Hindawi Publishing Corporation, New York, 2006.

[5] V. Lakshmikantham, D. Bainov, P. Simeonov, Theory of Impulsive Differential Equations, World Scientific, Singapore, 1989.

[6] A.M. Samoilenko, N.A. Perestyuk, Impulsive Differential Equations, World Scientific, Singapore, 1995.

[7] R.P. Agarwal, M. Benchohra, D. O'Regan, and A. Ouahab, Second order impulsive dynamic equations on time scales, Functional Differential Equations 11 (2004), 223-234.

[8] M. Benchohra, S.K. Ntouyas, A. Ouahab, Existence results for second order boundary value problem of impulsive dynamic equations on time scales, J. Math. Anal. Appl. 296 (2004), 65-73.

[9] M. Benchohra, S.K. Ntouyas, A. Ouahab, Extremal solutions of second order impulsive dynamic equations on time scales, J. Math. Anal. Appl. 324 (2006), 425-434.

[10] F.T. Fen, I.Y. Karaca, Existence of positive solutions for nonlinear second-order impulsive boundary value problems on time scales, Mediterr. J. Math. 13 (2016), 191-204.

[11] F.T. Fen, I.Y. Karaca, Positive solutions for a fourth-order multipoint impulsive BVP with p-Laplacian on time scales, Dyn. Sys. Appl. 7 (2016), 83-89.

[12] J. Henderson, Double solutions of impulsive dynamic boundary value problems on a time scale, J. Difference Equ. Appl. 8 (2002), 345-356.

[13] I.Y. Karaca, F.T. Fen, Multiple positive solutions for nonlinear second-order m-point impulsive boundary value problems on time scales, Filomat 29 (2015), 817-827.

[14] I.Y. Karaca, F.T. Fen, On positive solutions of nonlinear third-order impulsive boundary value problems on time scales, Mediterr. J. Math. 13 (2016), 4447-4461.

[15] I.Y. Karaca, F.T. Fen, Existence of positive solutions for nonlinear third-order m-point impulsive boundary value problems on time scales, Ukrainian Math. J. 68 (2016), 458-474.

[16] O.B. Ozen, I.Y. Karaca, F. Tokmak, Existence results for p-Laplacian boundary value problems of impulsive dynamic equations on time scales, Adv. Difference Equ. 334 (2013), 1-14.

[17] F. Tokmak, I.Y. Karaca, Positive solutions of an impulsive second-order boundary value problem on time scales, Dyn. Contin. Discrete Impuls. Syst. Ser. A Math. Anal. 20 (2013), 695-708.

[18] F. Tokmak, I.Y. Karaca, Existence of positive solutions for p-Laplacian impulsive boundary value problems on time scales, J. Inequal. Appl. 196 (2014), 1-14. 
[19] İ. Yaslan, Existence of positive solutions for nonlinear three-point problems on time scales, J. Comput. Appl. Math. 206 (2007), 888-897.

[20] I.Y. Karaca, O.B. Ozen, F. Tokmak, Multiple positive solutions of boundary value problems for p-Laplacian impulsive dynamic equations on time scales, Fixed Point Theory 15 (2014), 475-486.

[21] F.T. Fen, I.Y. Karaca, On positive solutions of m-point boundary value problems for p- Laplacian impulsive dynamic equations on time scales, Indian J. Pure Appl. Math. 46 (2015), 723-738.

[22] D.R. Smart, Fixed Point Theorems, Cambridge University Press, 1980.

[23] R.I. Avery, J. Henderson, Two positive fixed points of nonlinear operators on ordered Banach spaces, Commun. Appl. Nonlinear Anal. 8 (2001), 27-36.

[24] R.I. Avery, A generalization of the Legget-Williams fixed point theorem, Math. Sci. Res. Hotline 2 (1998), 9-14. 